\title{
Gastric schwannoma with giant ulcer and lymphadenopathy mimicking gastric cancer: a case report
}

\author{
Caihua Tang ${ }^{1 * \dagger} \mathbb{D}$, Qiyong Pan ${ }^{1 \dagger}$, Zeqing $\mathrm{Xu}^{1}$, Xuan Zhou ${ }^{2}$ and Ying Wang ${ }^{1}$
}

\begin{abstract}
Background: Gastric schwannomas are rare benign tumors originating from the intramuscular plexus of the stomach and account for just 2.6\% of gastric mesenchymal tumors. Gastric schwannoma (GS) with a surface ulcer is very rare. Herein, we report a rare case of an ulcer-bearing GS, which in conjunction with multiple enlarged regional lymph nodes, readily mimicked gastric cancer (GC).

Case presentation: A 79-year-old female presented with poor appetite and intermittent vomiting of gastric contents during the past month. Gastroscopy revealed a giant crateriform ulcer within the stomach body (at the angular notch). Its raised and indurated border was fragile and bled easily. GC was thus suspected. Contrastenhanced computer tomography $(\mathrm{CT})$ revealed a mild enhancement of the corresponding irregularly thickened gastric wall, and an annular zone of mucosal discontinuity. Enlarged regional lymph nodes were also found, making GC with metastases of lymph nodes our primary concern. ${ }^{18}$ F-fluorodeoxyglueose position emission tomography $\left({ }^{18} \mathrm{~F}-\mathrm{FDG} \mathrm{PET}\right) / \mathrm{CT}$ was then performed for further staging. Obviously increased FDG uptake was shown in the gastric lesion ((maximum standardized uptake value (SUV $\max )$ 14.6), but no FDG uptake was observed in the enlarged regional lymph nodes. Given the strong suspicion of GC, subtotal gastrectomy was performed. GS was revealed by postoperative pathology, with no evidence of metastasis in the 13 resected lymph nodes.

Conclusions: This was a rare case of GS with a giant surface ulcer and multiple enlarged regional lymph nodes. The uptake of ${ }^{18} \mathrm{~F}-\mathrm{FDG}$ in the tumor was substantially higher than previously published literature reports. Under these circumstances, it is difficult to be differentiated from GC.
\end{abstract}

Keywords: Gastric schwannoma, Positron emission tomography, FDG, Ulcer

\section{Background}

Schwannoma is a benign tumor originating from the nerve sheath that rarely involves the digestive tract. Gastric schwannomas arise from the intramuscular plexus of the stomach wall, accounting for just $2.6 \%$ of gastric mesenchymal tumors and only $0.2 \%$ of gastric neoplasms [1]. Herein, we report a rare case of gastric schwannoma (GS) that mimicked gastric cancer (GC). A giant surface ulcer and resultant lymphadenopathy created a false impression of malignancy.

\footnotetext{
* Correspondence: tcaihua2004@126.com

${ }^{+}$Caihua Tang and Qiyong Pan contributed equally to this work.

1 Department of Nuclear Medicine, The Fifth Affiliated Hospital, Sun Yat-sen

University, Zhuhai 519000, China

Full list of author information is available at the end of the article
}

\begin{abstract}
${ }^{18} \mathrm{~F}$-fluorodeoxyglueose position emission tomography $\left({ }^{18} \mathrm{~F}\right.$-FDG PET) has been used extensively for the differential diagnosis of benign or malignant tumors and the FDG uptake has been demonstrated to have a significant correlation with the malignant potential of various tumors [2]. ${ }^{18}$ F-FDG PET imaging of GS has only been reported in a few cases, all demonstrating increased FDG uptake ranging from $\mathrm{SUV}_{\max }$ of 3.3 to 7.1 [3-7]. In our patient, the tumor showed an obviously increased FDG uptake $\left(\mathrm{SUV}_{\max }\right.$ 14.6), which was significantly higher than that of other cases reported in the literature.
\end{abstract}

\section{Case presentation}

A 79-year-old female presented with poor appetite and intermittent vomiting of gastric contents during the past month. A regimen of proton pump inhibitors offered no

(c) The Author(s). 2020 Open Access This article is distributed under the terms of the Creative Commons Attribution 4.0 International License (http://creativecommons.org/licenses/by/4.0/), which permits unrestricted use, distribution, and 
major symptomatic relief. Gastroscopy revealed a sizeable $(4.5 \mathrm{~cm} \times 6.0 \mathrm{~cm})$ crateriform ulcer within the body of the stomach, at the angular notch. The raised, indurated border was fragile and bled easily. Thus, GC was suspected. Histopathology after biopsy showed inflammatory granulation tissue, exudation and necrotic material, and no malignant tumor cells were found. Contrast-enhanced computed tomography (CT) of the upper abdomen showed a mild enhancement of the gastric body, accentuating an irregularly thickened wall. An annular zone of mucosal discontinuity was also identified (Fig. 1 b), and a number of enlarged lymph nodes were noted in the vicinity (Fig. $1 \mathrm{~d}$ ), the largest one measuring $12 \mathrm{~mm} \times 9 \mathrm{~mm}$. Malignancy was strongly suspected, either GC with multiple nodal metastases or lymphoma.

Routine blood, urine and stool tests were all within normal ranges, as were indices of liver and kidney function. Serum assays for various tumor markers were negative. ${ }^{18} \mathrm{~F}-\mathrm{FDG}$ PET/CT was performed for further staging purposes. The images disclosed a protuberant soft tissue mass in the gastric cavity, with a heterogeneous increase in FDG uptake (maximum standardized uptake value $\left(\mathrm{SUV}_{\max }\right)$ 14.6). However, no FDG uptake was observed on the enlarged perigastric lymph nodes (Fig. $1 \mathrm{~d}$ ). Malignancy was still our chief concern, especially GC, although the weak nodal uptake suggested a reactive process.

Subtotal laparoscopic gastrectomy was subsequently performed. GS was revealed by postoperative pathology. Histopathology revealed a mass with necrosis on the surface. The lesion was sallow, firm, and deeply entrenched in the muscular layer, without breaching serosa. Hematoxylin and eosin (H\&E) stained slides revealed abundant spindle cells. The Ki-67 protein level, reflecting cellular proliferative activity, was $<5 \%$. Immunohistochemical examination showed that S-100 and SOX10 were positive (Fig. 2 b), whereas CK7, CK20, CK, villin, CDX-2, CD117, CD34, SMA, desmin, and c-erbb-2 were negative. No tumor metastases were seen in any of the sections of the excised lymph nodes (0/13). The patient has done well one year following surgery, showing no signs of recurrence or metastases.

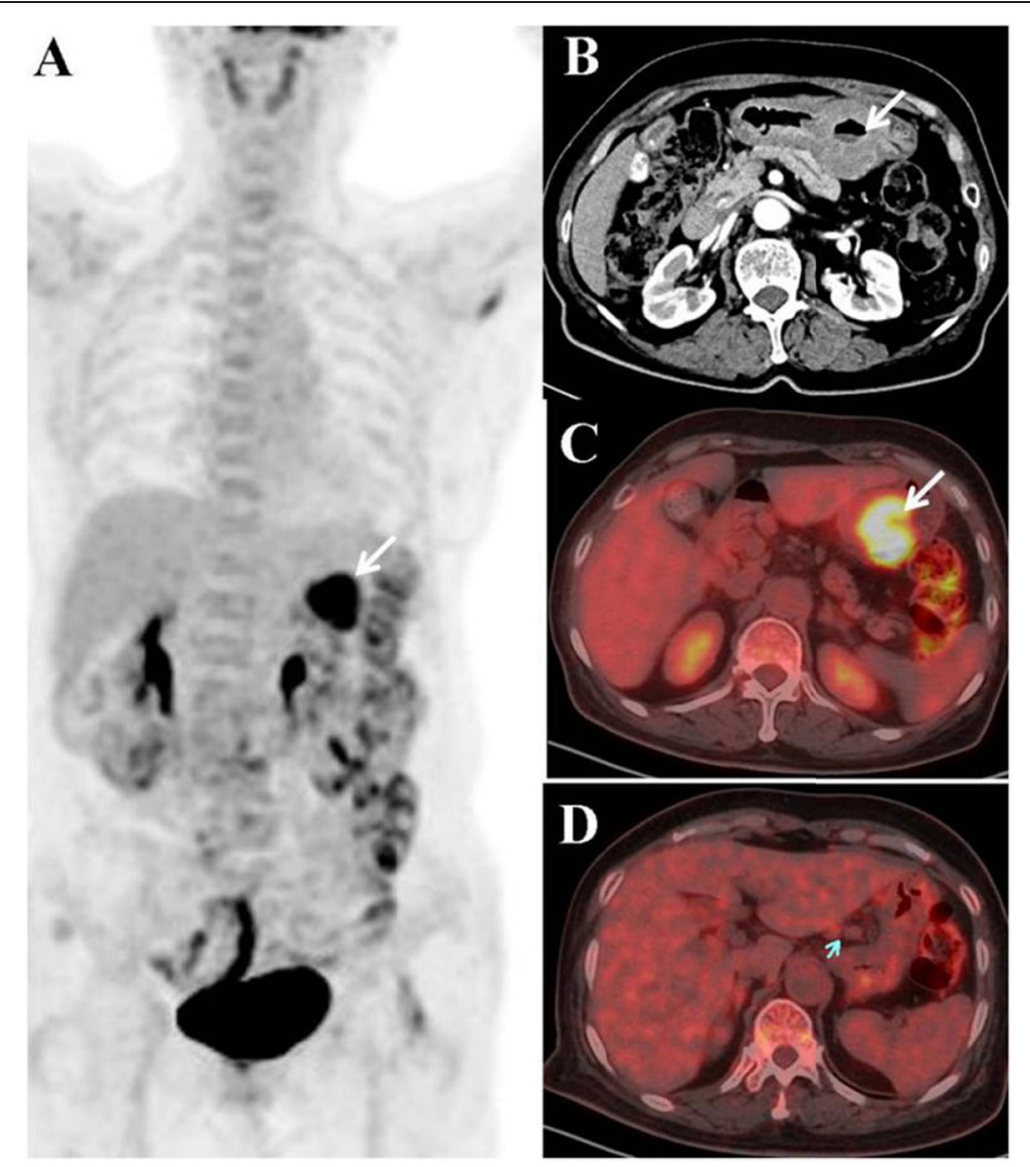

Fig. 1 Radiographic details $\mathbf{a}^{18}$ F-FDG PET shows a high accumulation of FDG in left upper abdomen (white arrow). b Contrast-enhanced CT view of the irregularly thickened gastric wall with slight enhancement. c Increased FDG uptake was observed in the gastric mass (SUV max 14.6 , white arrow). d Enlarged lymph nodes abutting the lesser curvature of the stomach, lacking FDG intensity (blue arrow) 


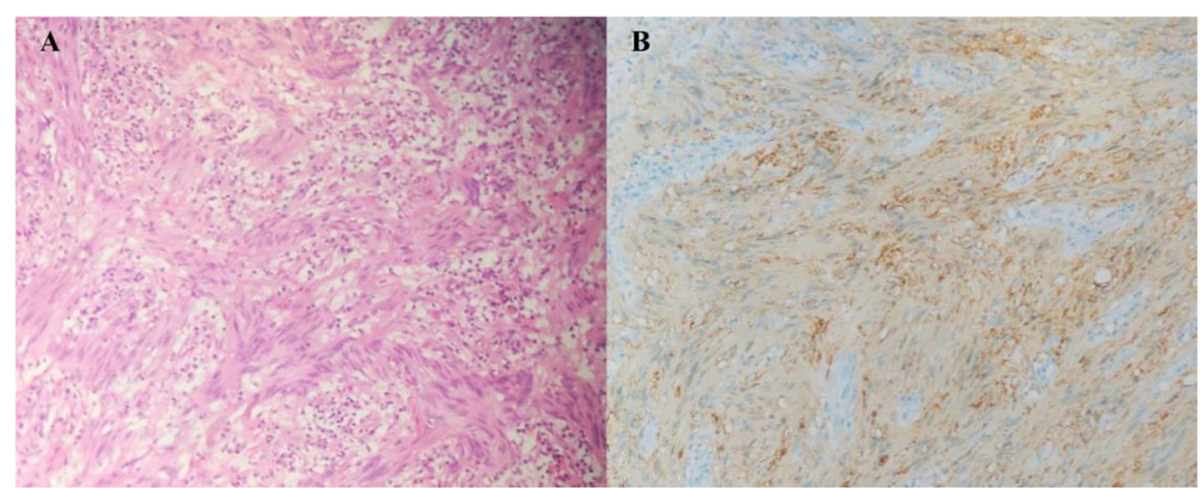

Fig. 2 Hispathological findings: a Spindled tumor cells with large nuclei and vague nuclear palisading (H\&E, $100 \times$ ). b The spindle tumor cells shows a positive reaction for S-100 protein by immunohistochemical staining $(100 \times)$

\section{Discussion and conclusion}

Schwannoma usually originates from the Schwann cell of the peripheral great nerve trunk, spinal nerve or cranial nerve, and rarely involves in the digestive tract. Gastric schwannomas are usually found in elderly women, and the most commonly referred symptom is abdominal discomfort, while upper gastrointestinal bleeding can be accompanied in patients with mucosal ulcer [8]. The tumors are usually benign, slow-growing, and have good prognosis [1]. In the present case, the patient has done well one year following surgery, showing no signs of recurrent or metastatic diseases.

GS must be differentiated from other submucosal tumors such as gastrointestinal stromal tumors (GISTs) [9], which originate from mesenchymal tissue as well. The locations, symptoms and growth patterns are frequently indistinguishable. The range of ${ }^{18} \mathrm{~F}$-FDG uptake in malignant GISTs is partly shared with that of GS. Nevertheless, necrosis and cystic degeneration are more common in the former, and local and distant metastasis can also be seen in malignant GISTs, while such features are very rare in GS. Mild to moderate, uniform, progressive enhancement is instead characteristic of GS [10], and enhancement of GISTs is generally higher than that of GS in contrastenhancement CT scans. A diagnosis of GS relies on histopathological examination. GISTs consist of spindled cells with nuclear palisading and may mimic schwannomas in particular. However, the immunohistochemical staining between these spindle cell tumors are different [11]. Positive CD34 and CD117 indicate GISTs, and positive S-100 indicates schwannomas. In the present case, the tumors revealed spindle cells, which were strongly positive for S-100 and SOX10 staining, and negative for CD117 and CD34, thus indicated the diagnosis of schwannomas.

At times, GS must be differentiated from GC, which often occurs at the lesser curvature of the stomach, creates an irregularly thickened wall, has indistinct borders, directy invades adjacent tissues, and spreads to lymph nodes [12].
Degrees of ${ }^{18} \mathrm{~F}$-FDG uptake partly overlapp in these two diseases, so the distinction was mainly based on CT morphology. However, distant metastasis and adjacent invasion can be detected by PET/CT, which helps us to differentiate and diagnose. This case was preoperatively misdiagnosed as $\mathrm{GC}$, mainly due to the irregular contours, extensive surface ulceration, and the remarkable intensity of ${ }^{18} \mathrm{~F}$-FDG uptake. The prominent perigastric lymph nodes, with weak ${ }^{18} \mathrm{~F}$ FDG uptake, may have reflected the extent of inflammation involved. Repeat deeper biopsy guided by endoscopic ultrasonography may be performd preoperatively, which was not considered because the ulcer border was fragile and bled easily. Maybe, it was the limitation of this case. We expect our case report would contribute to the recognition of the biological chracteristics of GS and to avoid inappropriate under- or overtreatment of the patients in the future.

${ }^{18}$ F-FDG PET/CT has been used extensively for the detection, staging, therapeutic monitoring, and follow-up of a variety of malignant tumors [2]. The FDG uptake has been demonstrated to have a significant correlation with the malignant potential of GISTs [9]. To the best of our knowledge, only a few cases of gastric schwannoma have been reported with ${ }^{18} \mathrm{~F}$-FDG PET/CT. Komatsu et al. [5] were the first to report a patient with GS who exhibited increased FDG uptake. Yap et al. [6] described a patient with histologically confirmed GS and nonHodgkin's lymphoma. ${ }^{18} \mathrm{~F}-\mathrm{FDG}$ PET/CT was performed before and after treatment for lymphoma, showing an increased uptake within the gastric lesion $\left(\mathrm{SUV}_{\max } 4.9\right)$. Takeda et al. [7] reported a patient with GS at the greater curvature of the body of the stomach. The lesion was rounded, showing inward growth, smooth edges, uniform density, and progressive enhancement in CT images. Increasing uniformity of ${ }^{18}$ F-FDG uptake (SUVmax 6.28) was observed by PET/CT. In our patient, FDG uptake $\left(\mathrm{SUV}_{\max } 14.6\right)$ by the tumor was significantly higher than that of other cases reported in the literature. This value also far exceeds the $\mathrm{SUV}_{\max }$ cutpoint of 6.1 
established by Benz et al. [13] for separating malignant from benign peripheral nerve sheath tumors (PNSTs). However, the benign histopathology features of the tumor in our patient refutes their stated threshold. Therefore, these data suggest that ${ }^{18} \mathrm{~F}$-FDG PET/CT has a limited role in making preoperative differential diagnosis between benign schwannoma and malignancy.

Schwannomas originating in the stomach are seldom associated with ulceration. Oh et al. [14] identified a GS within the submucosa of the lesser curvature of the stomach by gastroscopy, which with a central ulcer was misdiagnosed as a gastric malignant stromal tumor. Increased uptake of ${ }^{18}$ F-FDG (SUV ${ }_{\max } 7.1$ ) of the tumor was found by PET/CT. In the present case, a giant $(4.5$ $\mathrm{cm} \times 6.0 \mathrm{~cm}$ ) crateriform ulcer of the stomach at the angular notch was shown by gastroscopy, and tumor uptake of FDG was inordinately high, implying a high risk GC. However, the weak uptake shown by enlarged regional lymph nodes attested to be a reactive process. Although GS is a benign tumor, the FDG uptake of GS was significantly increased, which may be explained by the observed tumor influx of a number of inflammatory cells and the active expression of glucose transporter 3 and 1 in the tumor cells [15]. Further studies are needed to confirm the ${ }^{18}$ F-FDG uptake mechanism of GS.

In conclusion, this is a rare case of GS with giant surface ulceration and enlarged regional lymph nodes. The uptake of ${ }^{18}$ F-FDG by the tumor was significantly higher than levels previously reported in the literature. Ordinarily, GS must be differentiated from GIST, with GC becoming an added concern if surface ulceration and nodal enlargement are present.

\section{Abbreviations}

CT: Computer tomography; FDG: Fluorodeoxyglucose; GC: Gastric cancer; GISTs: Gastrointestinal stromal tumors; GS: Gastric schwannoma;

MIP: Maximum intensity projection; PET: Positron emission tomography; PNSTs: Peripheral nerve sheath tumors; SUV $\max$ : Maximum standardized uptake value

\section{Acknowledgments}

We would like to thank the patient for giving consent for publication, and we grateful for all research staff and co-investigators involved in this case study.

\section{Authors' contributions}

TCH made substantial contributions to the case report and research work. The initial draft of the manuscript was co-written by TCH and PQY. ZX expended much effort in acquiring clinical data, histopathologic findings and interpretative report. XZQ and WY were tasked with important intellectual content revisons. All authors have read and approved the final manuscript.

\section{Funding}

This work was supported in part by the National Natural Science Foundation of China (No. 81701723).

\section{Availability of data and materials}

To protect the patient's privacy, images and data of the current study are not publicly available.

\section{Ethics approval and consent to participate}

The patient provided written consent for the use of images and data for research purpose. As it is a case report, ethics approval is not necessary after consulting the Ethics Committee of the Fifth Affiliated Hospital, Sun Yat-sen University

\section{Consent for publication}

Written consent for publication has been granted by the patient, who agreed that details/images may be viewed on the Internet and accessed by the general public.

\section{Competing interests}

The authors declare that they have no competing interests..

\section{Author details}

'Department of Nuclear Medicine, The Fifth Affiliated Hospital, Sun Yat-sen University, Zhuhai 519000, China. ${ }^{2}$ Department of pathology, The Fifth Affiliated Hospital, Sun Yat-sen University, Zhuhai 519000, China.

Received: 21 October 2019 Accepted: 10 February 2020

Published online: 14 February 2020

\section{References}

1. Kinsey-Trotman S, Balalis G, Gupta A, Worley P. Gastric schwannoma [J]. J Gastrointest Surg. 2016;20(9):1666-8.

2. Bomanji JB, Costa DC, Ell PJ. Clinical role of positron emission tomography in oncology. Lancet Oncol. 2001;2:157-64.

3. Ohno T, Ogata K, Kogure N, Ando H, Aihara R, Mochiki E, et al. Gastric schwannomas show an obviously increased fluorodeoxyglucose uptake in positron emission tomography: report of two cases. Surg Today. 2011;41: 1133-7.

4. Hong K, Kim DY. F-18 FDG PET/CT of a gastric Schwannoma. Nucl Med Nucl Med Mol Imaging. 2011;45:238-40.

5. Komatsu D, Koide N, Hiraga R, Furuya N, Akamatsu T, Uehara T, et al. Gastric schwannoma exhibiting increased fluorodeoxyglucose uptake. Gastric Cancer. 2009;12:225-8.

6. Yap J, Huang YT, Lin M. Detection of synchronous gastric schwannoma on FDG PET/CT aided by discordant metabolic response [J]. Clin Nucl Med. 2015;40(5):e287-9.

7. Takeda M, Amano Y, Machida T, Kato S, Naito Z, Kumita S. CT, MRI, and PET findings of gastric schwarmoma [J]. Jpn J Radiol. 2012;30(7):602-5.

8. Lyros O, Schickel S, Schierle K, Hoffmeister A, Gockel I. Gastric schwannoma: rare differential diagnosis of acute upper gastrointestinal (Gl) bleeding. Z Gastroenterol. 2017:55(8):761-5.

9. Kamiyama $Y$, Aihara R, Nakabayashi T, Mochiki E, Asao T, Kuwano H, et al. ${ }^{18}$ F-Fluorodeoxyglucose positron emission tomography: useful technique for predicting malignant potential of gastrointestinal stromal tumors. World J Surg. 2005;29:1429-35.

10. Ji JS, Lu CY, Mao WB, Wang ZF, Xu M. Gastric schwannoma: CT findings and clinic pathologic correlation [J]. Abdom Imaging. 2015;40(5):1164-9.

11. Fletcher CD, Berman JJ, Corless C, Gorstein F, Lasota J, Longley BJ, et al. Diagnosis of gastrointestinal stromal tumors: a consensus approach. Int J Surg Pathol. 2002;10:81-9.

12. Mochiki E, Kuwano H, Katoh H, Asao T, Oriuchi N, Endo K. Evaluation of ${ }^{18} \mathrm{~F}$ 2-deoxy-2-fluoro-D-glucose positron emission tomography for gastric cancer. World J Surg. 2004;28:247-53.

13. Benz MR, Czernin J, Dry SM, Tap WD, Allen-Auerbach MS, Elashoff D, et al. Quantitative F-18-fluorodeoxyglucose positron emission tomography accurately characterizes peripheral nerve sheath tumors as malignant or benign. Cancer. 2010;116:451-8.

14. Oh SJ, Suh BJ, Park JK. Gastric schwannoma mimicking malignant gastrointestinal stromal tumor exhibiting increased fluorodeoxyglucose uptake [J]. Case Rep Oncol. 2016;9(1):228-34.

15. Shimada Y, Sawada S, Hojo S, Okumura T, Nagata T, Nomoto K, et al. Glucose transporter 3 and 1 may facilitate high uptake of ${ }^{18} \mathrm{~F}$-FDG in gastric schwannoma. Clin Nucl Med. 2013;38:e417-20.

\section{Publisher's Note}

Springer Nature remains neutral with regard to jurisdictional claims in published maps and institutional affiliations. 